\title{
Macro- and Microscopic Study of "Smart Water" Flooding in Carbonate Rocks-An Image-based Wettability Examination
}

\section{Supplemental Information}

Hongna Ding, Yuzhu Wang, Arthur Shapoval, Yuyun Zhao and Sheik Rahman*

School of Minerals and Energy Resources Engineering, University of New South Wales, Sydney, NSW 2052, Australia

sheik.rahman@unsw.edu.au

\section{Tomo images of Austin chalk AC4b by $\mu-C T$ scanning}

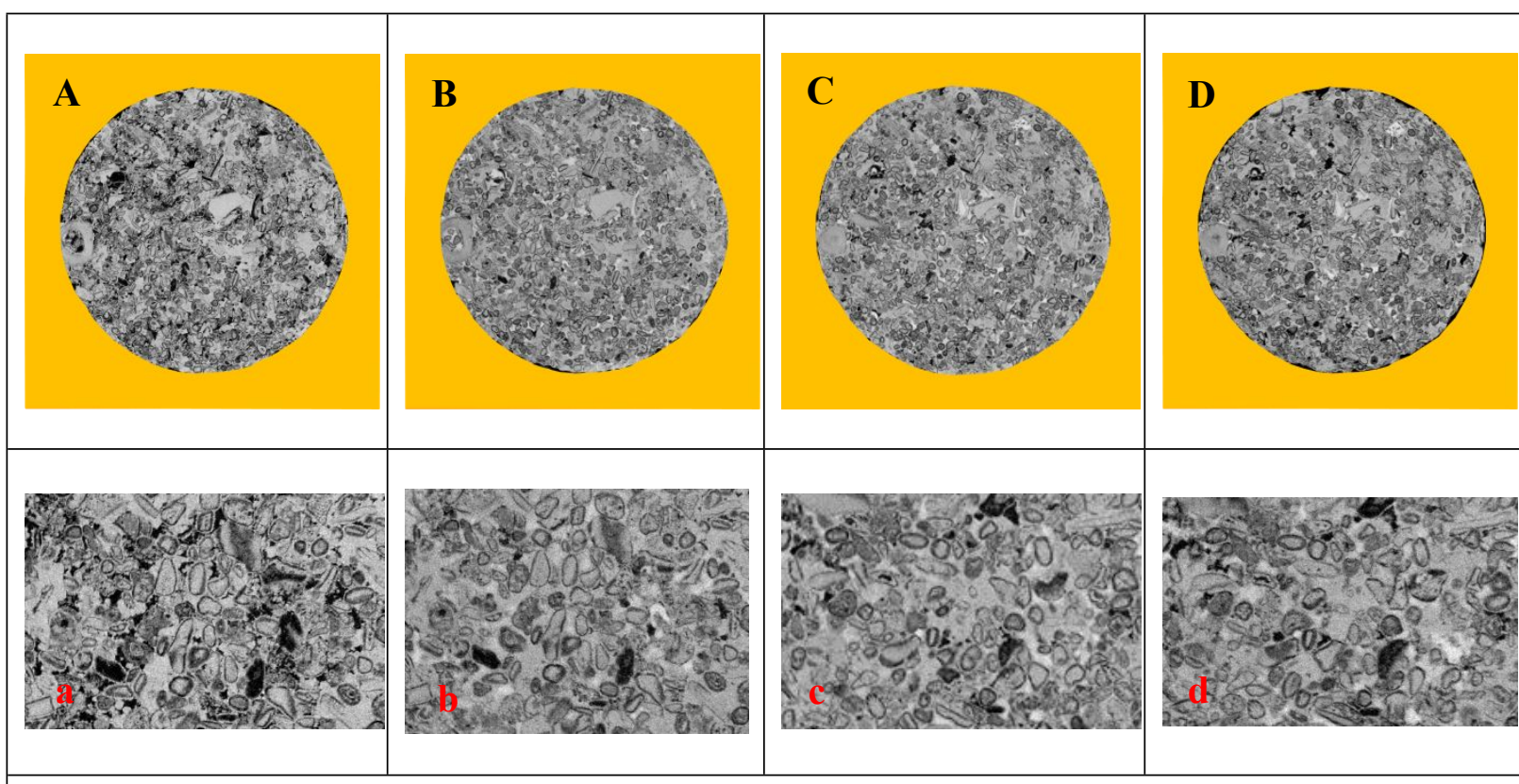

Fig. S1. Tomo images of Austin chalk AC4b scanned with a resolution of $3.0 \times 3.0 \times 3.0 \mu \mathrm{m}^{3} / \mathrm{voxel}$. (A) is the image of the dry sample; (B) is the image of the sample after aging; $(\mathbf{C})$ is the image of the sample after SW flooding; (D) is the image of the sample after SW0.25Ca flooding. Images (a) to (d) present the subset 


\section{Pressure curves in the core flooding experiments}

It can be seen from Figs. S2 and S4 that the pressure drop (dP) in SW flooding increases quickly to 4 psi by injecting 2 and 4 pore volumes (PV) of SW for chalk AC4a and AC4b, respectively, while the $\mathrm{dP}$ value remains nearly unchanged in the later injection period, e.g., after 4.0 PV for chalk AC4a and 9.0 PV for chalk AC4b. In direct contrast to the SW cases, it is found that $\mathrm{dP}$ in SW4SO flooding increases to 6 psi after $6 \mathrm{PV}$ injection, followed by a short stable period from $6 \mathrm{PV}$ to $8 \mathrm{PV}$, and afterwards, dP decreases to 5 psi gradually by further injecting SW4SO to $13 \mathrm{PV}$. As discussed in the main text, the SW4SO is effective in improving the water-wetness of nanopores in comparison with SW; thus, the high dP value in SW4SO injection (6 psi, see Fig. S3) compared to SW injection can be attributed to the invasion of SW4SO to nanopores. In addition, the decrease in $\mathrm{dP}$ value from 6 psi to 5 psi in SW4SO flooding can be ascribed to the invasion of SW4SO from nanopores to micropores. The dP in SW0.25Ca flooding shows a gradual increase with PV up to $16 \mathrm{PV}$, however, the $\mathrm{dP}$ values are less than 3 psi (see Fig. S5). This can be explained by the fact that SW0.25Ca is effective in improving the water-wetness of micropores in comparison with SW; thus, the dP value in SW0.25Ca flooding is lower (3 psi) than that in SW flooding (5 psi). Moreover, with an gradual invasion of SW0.25Ca from large micropores to small micropores, the $\mathrm{dP}$ value will increase with the increase in PV.

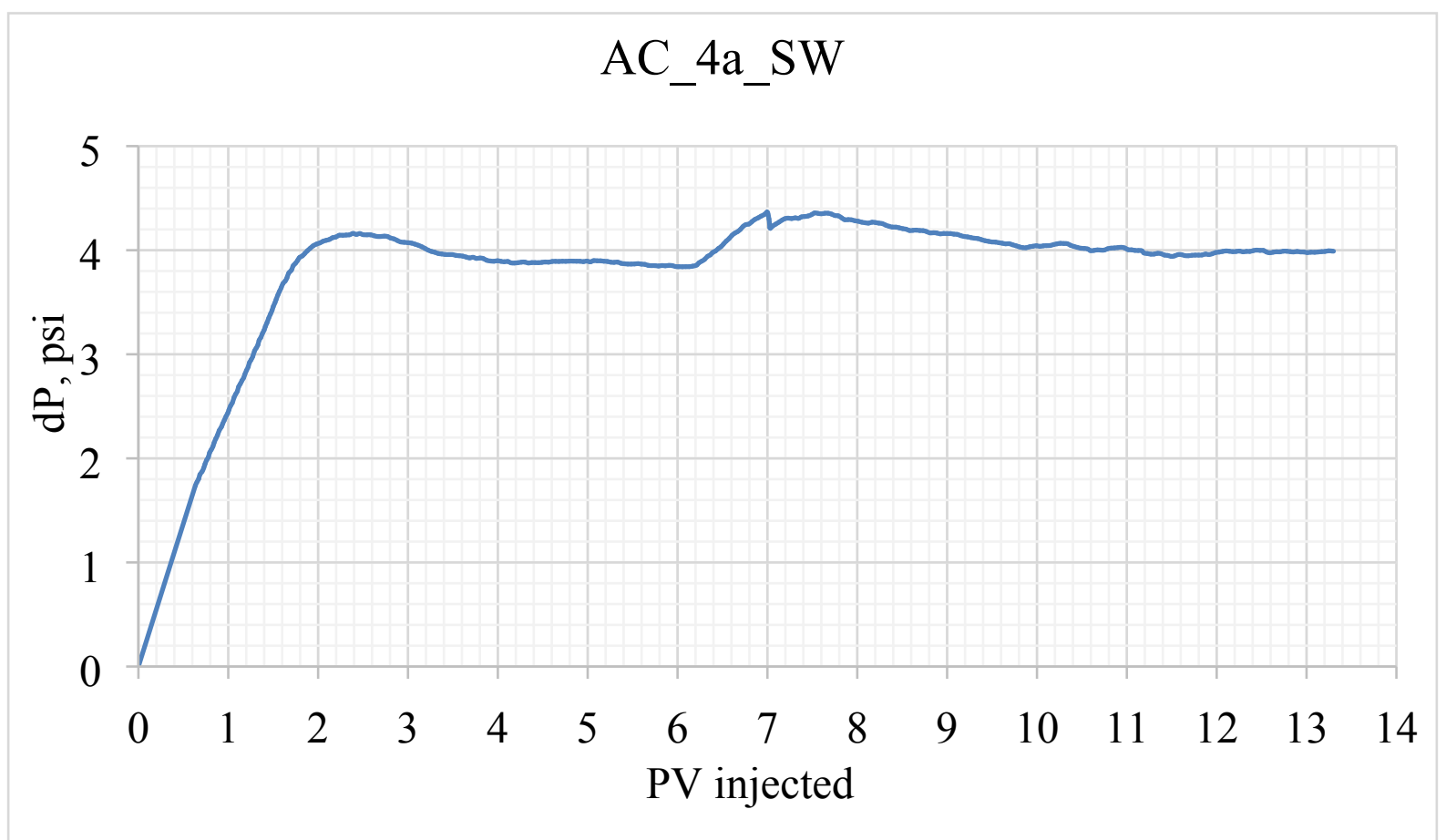

Fig. S2. Pressure curve of chalk AC4a with SW flooding. 


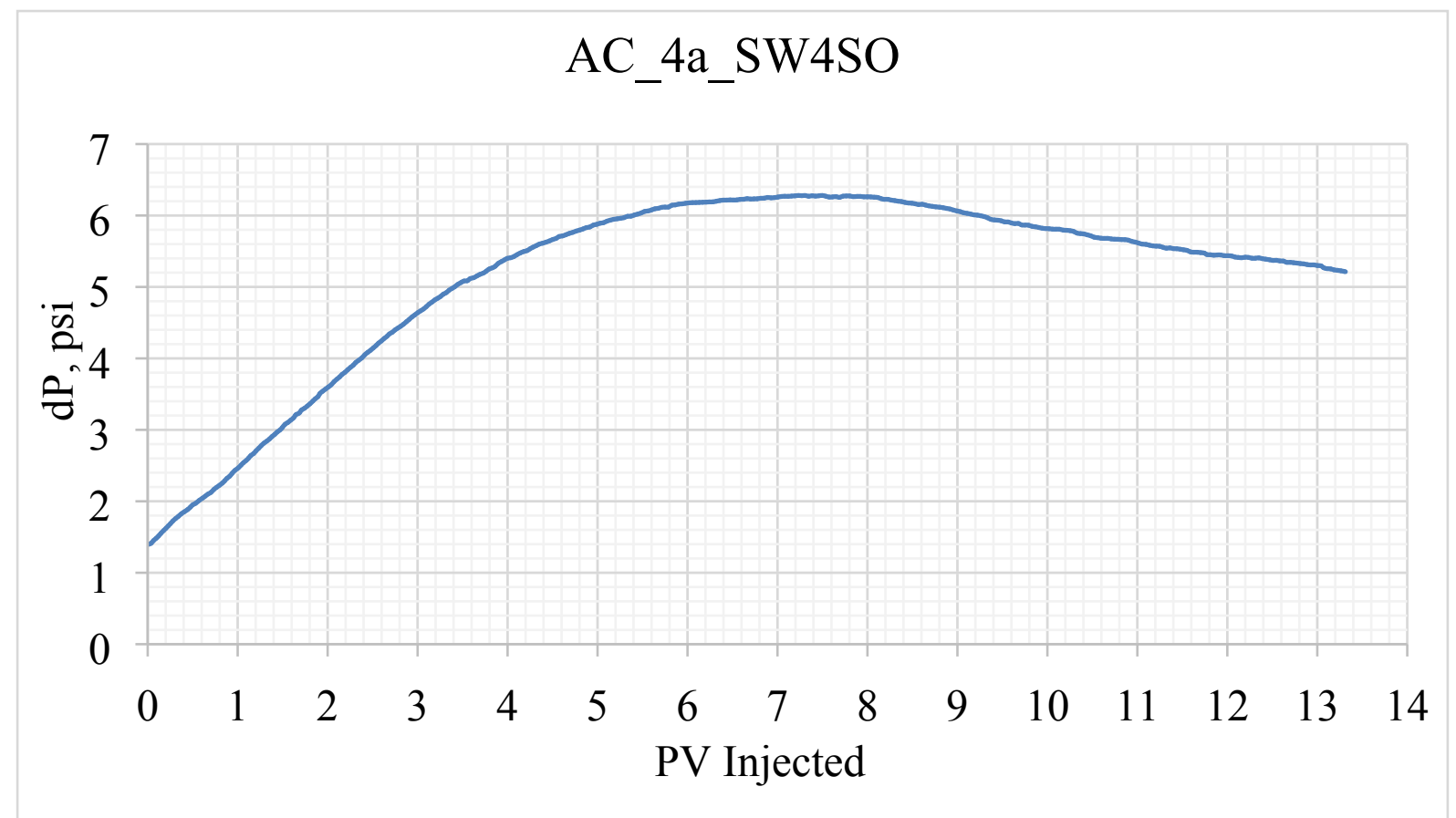

Fig. S3. Pressure curve of chalk AC4a with SW4SO flooding.

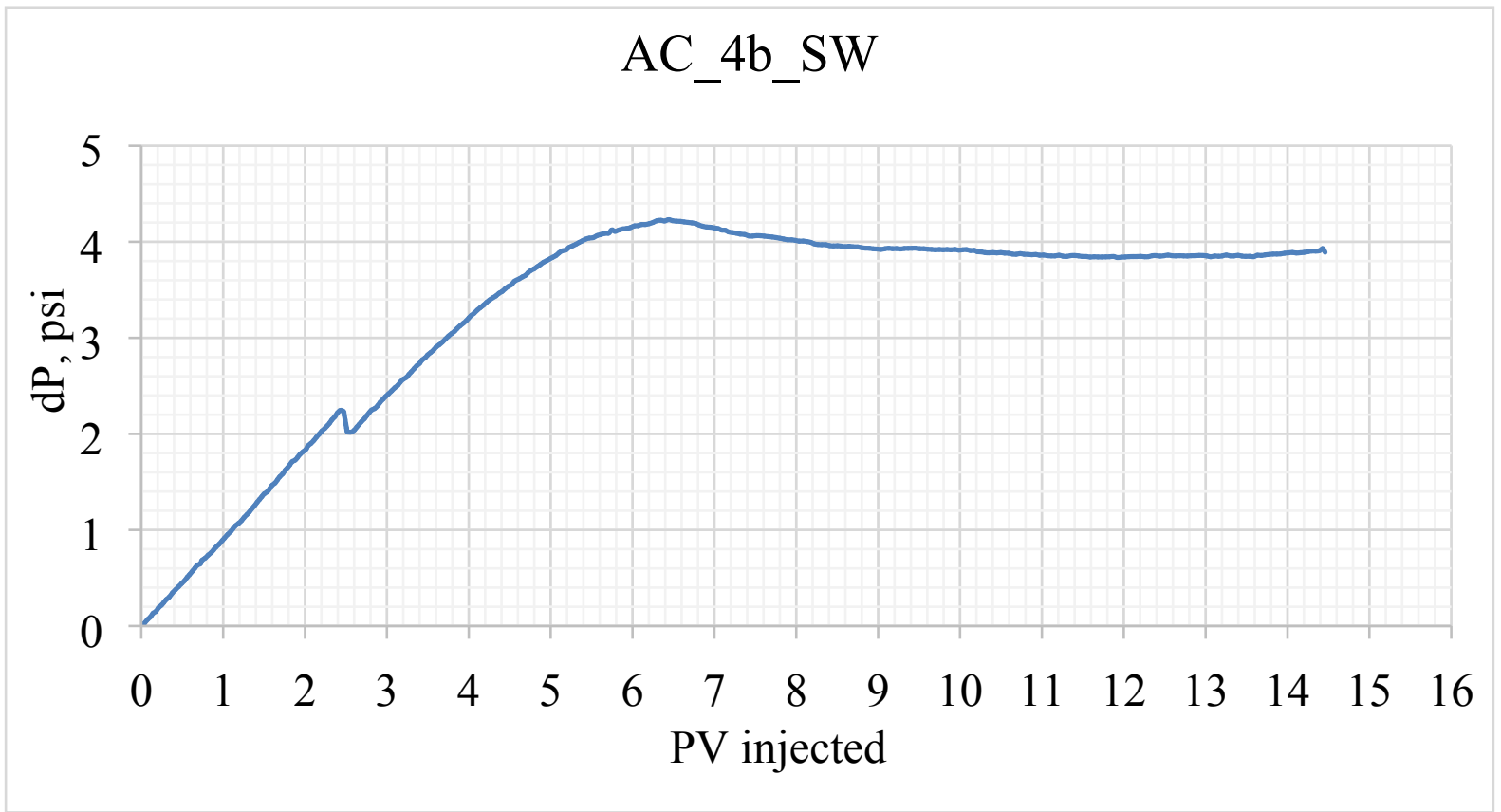

Fig. S4. Pressure curve of chalk AC4b with SW flooding. 


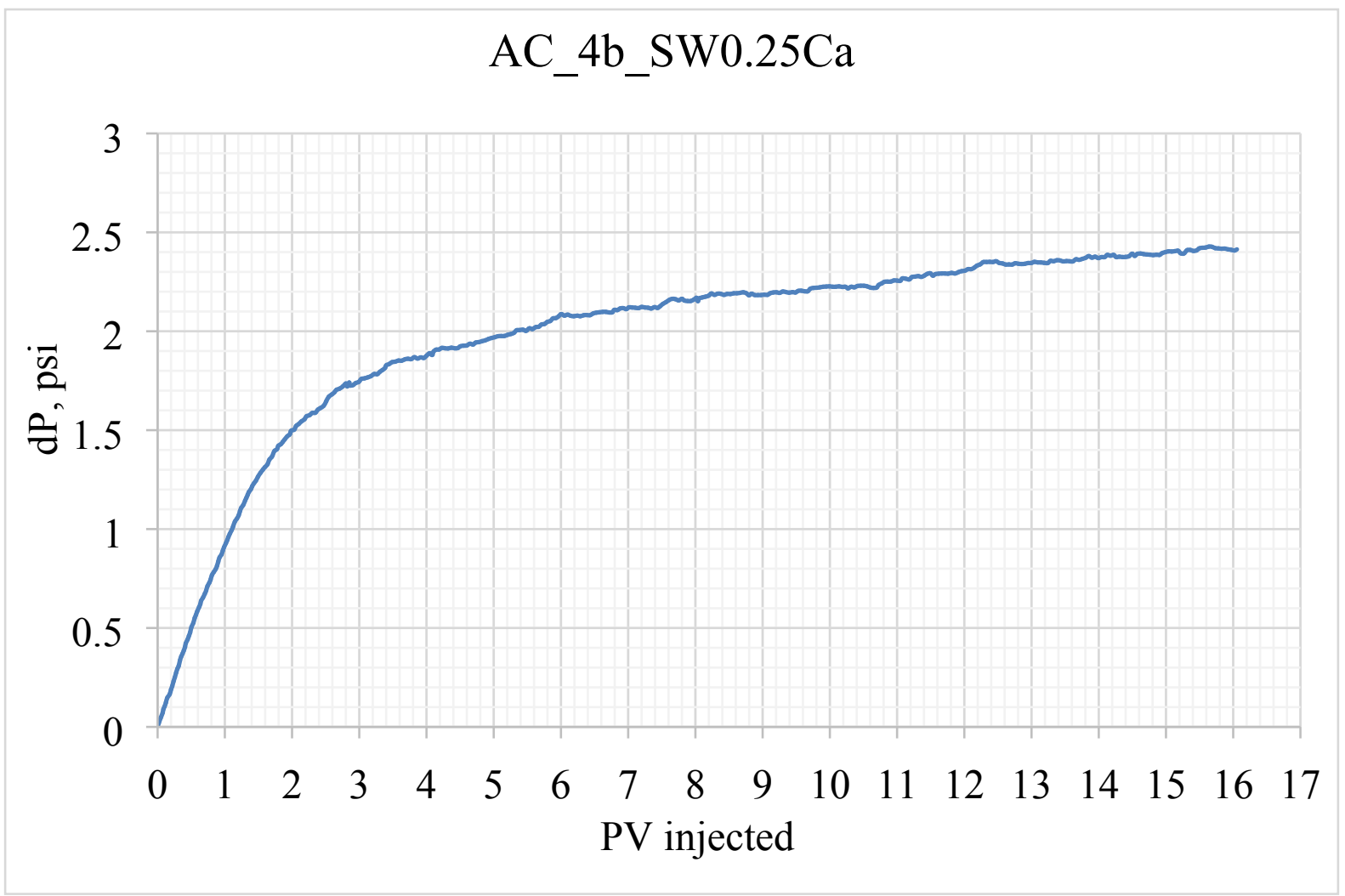

Fig. S5. Pressure curve of chalk AC4a with SW4SO flooding. 